\title{
Scientists can publish their best work at any age
}

New equation also suggests way to predict a researcher's potential to produce top work.

\section{Philip Ball}

03 November 2016

Hoping that your next paper will be the big one? It just might be — the chance that your next article will be your best-cited is as good as ever, no matter where you are in your career.

That's the finding of a team led by Albert-László Barabási at Northeastern University in Boston, Massachusetts. The researchers analysed the papers of thousands of scientists from different disciplines. Considering their publication records as a sequence of articles, the most highly-cited were equally likely to be found at the beginning, middle or end of the sequence ${ }^{1}$.

"We scientists are random," Barabási says. "Every time we publish a paper, we have the same chance of publishing our biggest hit as we do with any other paper."

This might seem to conflict with the well-documented finding that big discoveries and high-impact work tend to happen early in a scientist's career. But there's no contradiction, because the new work also shows that productivity - the number of papers produced per year - tends to slowly decline over a typical career. A scientist's chance of securing a 'greatest hit' accordingly decreases over time, simply because they have fewer shots at it.

Image: KimAlbrecht. Data Image: Roberta Sinatra.

Each circular line represents a scientists' career, where the bumps represent the impact of papers. If a career is regarded as a sequence of papers, then success is randomly distributed within the sequence, researchers say.

\section{Innate potential?}

But the researchers also make a more contentious calculation. They devise a simple mathematical model that describes the probability that any particular paper will be a hit. This depends on only two factors, they argue: an element of luck, and a certain quality, or $Q$ factor, that measures an individual scientist's ability to boost the impact of any project. 
Testing their model against the publication records of 2,887 physicists, the team found that the equation implies that the 'luck' factor is the same for all scientists. The $Q$ factor is obtained from a researcher's citation record: it is proportional to the logarithm of the number of citations that a scientist has received over a certain time frame.

The researchers anticipated that $Q$ would increase over the course of a scientific career, as an individual becomes more experienced. To their surprise, they found that it remains mostly constant.

That's shocking because it seems to imply that $Q$ — the multiplier that makes someone capable of capitalizing on luck to produce a big hit - is a quality that a scientist brings to their work at the outset, and which they cannot easily change thereafter.

"I hate to call it innate," Barabási says, "but it seems to be a combination of your ability and education. Once you start your career, you have it and it stays with you."

And it does seem to carry predictive value: high $Q$ values pick out Nobel laureates and other prize-winning physicists more reliably than do other metrics, such as the widely-used $h$-index, which measures cumulative productivity and the impact of publications. (A scientist with an $h$-index of 20 has published 20 papers, each of which has at least 20 citations.

$Q$ is predictive even early in a scientific career, the team says. Calculating a scientist's $Q$ value after they have published 20 papers enables accurate prediction of what their $h$-index will be after 40 papers for $74 \%$ of researchers studied.

But a high $Q$ alone doesn't guarantee a glittering career. Scientists need to sustain their motivation, too: the chance of writing a 'hit paper' depends not just on $Q$ and a degree of luck but also on productivity.

"The novelty here is that one can extract a measure of individual potential that could indicate whether or not someone will ever achieve greatness, if she has not already," says Santo Fortunato, a physicist who specializes in informatics at Indiana University Bloomington.

\section{Predicting impact}

Barabási and his colleagues have previously described a model that allows them to predict how many citations a paper will gain on the basis of its previous citation history ${ }^{2}$. But attempts to predict scientists' future performance have had mixed results.

$Q$, then, might be destined to join the group of indices — the $h$-index most prominently among them — by which scientists are ranked and potentially assessed.

Barabási has mixed feelings about whether that's a good thing, but says that it is up to the community to decide how $Q$ is applied. If metrics are to be used at all, he says, then diversity is good, not least because it can avoid over-dependence on a few metrics that might be applied beyond their limitations. "The h-index is not always being well used," he says.

“I wouldn't favour the idea of supporting only the highest- $Q$ scientists," says Anthony van Raan, who conducts quantitative studies of science at Leiden University in the Netherlands. But he admits that such preferential support "could be interesting in this time of so many scientists and restricted resources".

But perhaps, says Barabási, $Q$ is already a kind of selection factor, whereby individuals with a low $Q$ score drop out of an academic scientific career at an early stage. The challenge now, he feels, is to work out what determines $Q$. If education does indeed play a big part, then maybe the knowledge can be used to assess and improve the way scientists are trained.

Nature I doi:10.1038/nature.2016.20926

\section{References}

1. Sinatra, R., Wang, D., Deville, P., Song, C. \& Barabási, A.-L. Science 354, aaf5239 (2016).

2. Wang, D., Song, C. \& Barabási, A-L., Science 342, 127-132 (2013). 